\title{
Correlation between charge carriers mobility and nano morphology in a blend of P3HT/PCBM bulk Heterojunction solar cell: Impact on recombination mechanisms
}

\section{O. Oklobia and T. S. Shafai}

Thin Films Laboratory, Faculty of Computing, Engineering, and Science, Staffordshire University, Sciences Centre, Leek Road, Stoke-on-Trent, ST4 2DF. UK.

Sandwich configurations consisting of hole-only and electron-only devices are employed to study the transport properties of polymer/fullerene blended material. Upon thermal annealing at $150{ }^{\circ} \mathrm{C}$ and subsequent device characterisations a direct correlation between solar cell device power conversion efficiency and charge carrier mobilities is identified. The highest power conversion efficiency reported herein resulted from the electron to hole mobility ratio of 4.78 . This ratio further reduces to 0.357 at $175^{\circ} \mathrm{C}$ as a result of the deterioration in electron mobility at the same time as enhancement in hole carrier mobility with no further improvement in PCE. Effects of thermal annealing on trap density, reveals that the trap assisted recombination is more dominant as compared to the bimolecular recombination. Furthermore such a mechanism enhances as a result of increasing trap density at higher annealing temperatures. Effect of light intensity on the open circuit voltage $V_{O C}$ further verifies that the dominant recombination process is through trap assisted recombination.

Keywords: Charge carrier mobility, trap density, bimolecular recombination, trap-assisted recombination, open circuit voltage.

\section{Introduction}

Recent progress in material selections and fabrication processes have led to the development of organic solar cells with efficiencies approaching 10\% [1-4]. Whilst there have been extensive research based on the blend of P3HT/PCBM solar cell [5-8], no breakthrough in power conversion efficiencies (PCEs) above $10 \%$ has been realised as yet. The internal quantum efficiency is given by $\eta=\eta_{\mathrm{A}} \eta_{\mathrm{D}} \eta_{\mathrm{CT}} \eta_{\mathrm{CC}}$ [9], where $\eta_{A}$ is the efficiency associated with photon absorption, $\eta_{D}$ is the efficiency associated with exciton dissociation, $\eta_{\mathrm{CT}}$ is the efficiency associated with charge transfer and $\eta_{\mathrm{CC}}$ is efficiency associated with charge collections at respective electrodes. Enhancing $\eta_{A}$ is very much dependent on the material selected. Amongst blended materials, poly (3-hexylthiophene) (P3HT) and phenyl- $\mathrm{C}_{61}{ }^{-}$ butyric acid methyl ester (PCBM) are currently the most investigated [10-12]. This is attributed to the high degree of crystallinity of P3HT and its absorption spectrum extending in the region of $650 \mathrm{~nm}$, and suitable electronic band gap favouring efficient charge transfer [13]. When considering $\eta_{D}$ and 
$\eta_{C C}$, again material selection is a significant factor concerning efficient charge separations resulting from differences in the lowest unoccupied molecular orbitals (LUMO) of the blended materials and charge transport properties. Furthermore donor/acceptor interfaces, nanodomain, transport pathways to the respective electrodes, charge carrier mobilities, loss mechanisms via recombination etc. all have an impact on the device power conversion efficiencies [14-18]. Owing to the processing conditions of these blends, PCEs can remarkably improve upon subjecting the device to various physical and chemical processes [19-22]. Previously [23] we reported on the PCBM cluster formation resulting from different thermal annealing strategies and its impact on photocurrent generation. Nanostructuring of the photo active layer significantly influences charge carrier mobilities, charge separation and recombination processes, all of which have a direct impact on power conversion efficiencies. It is known that the charge carrier mobilities enhances exciton dissociations and charge carrier recombination which are believed to be a competing process [24]. This parameter can improve or deteriorate upon thermal annealing. To address the influence of trap density on charge carrier mobility, we suggest a range of thermal annealing temperatures above the glass transition temperature of $\mathrm{P} 3 \mathrm{HT}$, for this phenomenon to be studied. It is well established that the best device efficiency reported for $\mathrm{P} 3 \mathrm{HT} / \mathrm{PCBM}$ blend is at annealing temperature of $\sim 140^{\circ} \mathrm{C}$ [25]. However, thermal annealing at this temperature results in restructuring of nanodomains which consequently has a direct impact on exciton dissociation as well as recombination processes, as a by-product of changes in the polymer/fullerene interface. This manuscript is therefore focused on the effects of thermal annealing on transport properties of solar cell devices based on blended materials (P3HT/PCBM) as well as establishing the processes supporting recombination mechanisms.

\section{Experimental}

P3HT (regioregularity of 96.6\%, Ossila Ltd.), and PCBM (Solenne BV, The Netherlands) were first dissolved in 1, 2-dichlorobenzene (DCB) separately and stirred for one hour at $\sim 45^{\circ} \mathrm{C}$. The two pristine solutions were then blended to make a solution of total concentration of $25 \mathrm{mg} / \mathrm{mL}$, a 1:1 weight-ratio of P3HT: PCBM. This was left to stir for another one hour before filtering through a $0.45 \mu \mathrm{m}$ PTFE filter. The filtered P3HT/PCBM blend solution was then left to stir for a period of $16-18$ hours at room temperature in a nitrogen-filled glove box $\left(<1 \mathrm{ppm} \mathrm{O}_{2}\right.$, and $\left.\mathrm{H}_{2} \mathrm{O}\right)$. Before device fabrication, ITO coated substrates $\left(\sim 10 \Omega / \mathrm{cm}^{2}\right)$ were first cleaned with deionised water, acetone, and isopropyl, sequentially in an ultrasonic bath for 10 minutes each. Cleaned substrates were then transferred to a nitrogen-filled glove box. This was followed by spin casting at $5000 \mathrm{rpm}$, a layer $(\sim 40 \mathrm{~nm})$ of poly (3, 4-ethylenedioxythiophene): poly (styrenesulfonate) (PEDOT: PSS) (Sigma Aldrich), onto the ITO 
coated substrate. PEDOT: PSS was filtered through a $0.45 \mu \mathrm{m}$ filter prior to spin casting. The PEDOT: PSS film was subjected to 10 minutes of baking at $\sim 150^{\circ} \mathrm{C}$. Following the cooling down of the substrate, P3HT/PCBM blend films were spun casted onto the PEDOT: PSS layer and left to dry in the desiccator. To complete the device fabrication, an aluminium (Al) layer ( $\sim 100 \mathrm{~nm})$ was thermally deposited onto the active layer through a shadow mask, under a vacuum pressure of $\sim 10^{-6}$ mbar. The device's active area, defined by a shadow mask is $0.14 \mathrm{~cm}^{2}$. To measure charge carrier mobilities, hole - and electron - only devices were fabricated. The hole - only device was fabricated following the same procedure as for the solar cell device above, but replacing the aluminium top electrode with gold $\mathrm{Au}$. And as for electron - only device, PEDOT: PSS was replaced with a thin layer of Cesium carbonate $\left(\mathrm{Cs}_{2} \mathrm{CO}_{3}\right)$, spun casted from solution in 2-ethoxyethanol.

The current-voltage (I-V) curves were measured with a Keithley 2400 source meter in the dark and under Air Mass 1.5 (AM 1.5), $100 \mathrm{~mW} / \mathrm{cm}^{2}$ illumination provided by an LOT Oriel 150-W solar simulator fitted with a Xenon lamp. For voltage dependence on light intensity measurements, the illumination intensity of the solar simulator was varied, by varying the power output of the lamp's power supply. The intensity of the illumination was checked every time before measurements with a calibrated Si reference solar cell and meter (Newport and Oriel Instruments). The thicknesses of the thin films were determined using a DektakXT stylus profiler. All thermal annealing of devices were done inside a nitrogen filled glove box. For hole - and electron - only devices, $I-V$ characterisations (for hole - and electron - only devices) were performed in the dark, whereas for solar cell device, this was done under illumination. All devices were tested inside a nitrogen filled glove box.

\section{Results and discussions}

\subsection{Implications of thermal annealing on bulk network transport properties}

Figure 1(a) represents current density/voltage characteristics for hole-only device fabricated using sandwich structure of ITO/PEDOT: PSS/P3HT: PCBM/Au. Upon positive application of voltage to Au contact with respect to PEDOT: PSS, there is no potential barrier and holes are injected from $\mathrm{Au}$ into the highest occupied molecular orbital (HOMO) of P3HT (reverse bias). This is illustrated in Fig. 2(a). At effective applied voltage $0.2 \mathrm{~V}<V_{\text {eff }}<0.7 \mathrm{~V}$ ( $V_{\text {eff }}$ being the applied bias corrected for the built-in potential arising from the difference between the work-function of contacts) the current density voltage obeys Ohm's law and the relationship may be written as

$J=q n_{\mathrm{e}(\mathrm{h})} \mu_{\mathrm{e}(\mathrm{h})} \frac{V}{d}$ 
where $q$ is the electron charge, $\mu_{\mathrm{e}(\mathrm{h})}$ is electron (hole) carrier mobility, $n_{\mathrm{e}(\mathrm{h})}$ is the electron (hole) concentration, and $d$ is the device active layer thickness. At higher effective applied field $3.5 \mathrm{~V}<V_{\text {eff }}$ $<4.4 \mathrm{~V}$ the current density voltage relationship departs from Ohmic behaviour and follows space charge limited current (SCLC). The current density dependence on voltage for trap filled limited SCLC is given by the square law

$J=\frac{9}{8} \varepsilon_{0} \varepsilon_{\mathrm{r}} \mu_{\mathrm{e}(\mathrm{h})} \frac{V^{2}}{d^{3}}$

where $\varepsilon_{0}$ represents permittivity of free space, $\varepsilon_{\mathrm{r}}$ is dielectric constant of the blended material $\sim 3.4$, $\mu_{\mathrm{e}(\mathrm{h})}$ is electron (hole) carrier mobility and $d$ is the device active layer thickness $\sim 150 \mathrm{~nm}$. Using the slope corresponding to the square law in Fig 1(a) and appropriate parameters, equation (2) yields a value of the hole mobility for as cast device as $\mu_{h}=1.15 \times 10^{-9} \mathrm{~m}^{2} \mathrm{~V}^{-1} \mathrm{~s}^{-1}$. This is in close agreement with published data for this parameter [26-27]. Similarly the Ohmic region of Fig1(a) together with appropriate parameters in equation (1) leads to a hole carrier concentration of $1.49 \times 10^{22} \mathrm{~m}^{-3}$ which is of the same order of magnitude reported in the literature [28]

At higher reverse applied field $V_{\text {eff }}>5 \mathrm{~V}$, the current density-voltage relationship is indicative of trapfilling states associated with exponent greater than 2 namely power law [29], which is given by:

$J=\left(\frac{\mu_{\mathrm{e}(\mathrm{h})} N_{\mathrm{CV}}}{q}\right)\left(\frac{\varepsilon_{0} \varepsilon_{\mathrm{r}}}{N_{\mathrm{T}_{\mathrm{e}(\mathrm{h})}}} \frac{l}{l+1}\right)^{l}\left(\frac{2 l+1}{l+1}\right)^{l+1} \frac{V^{l+1}}{d^{2 l+1}}$

where $N_{\mathrm{CV}}$ is the effective density of states in the conduction or valence band, $N_{\mathrm{T}_{\mathrm{e}(\mathrm{h})}}$ is the trap density for electrons (holes). Employing equation (3) yields $N_{\mathrm{T}_{\mathrm{h}}}$ of $2.98 \times 10^{25} \mathrm{~m}^{-3}$ for as cast device. For the sake of clarity the voltage regions for which the parameters are extracted are included in Table1. It is worth noting that the Ohmic region for both electron and hole devices are in the range $0-1 \mathrm{~V}$ whereas for space charge limited conductivity the range varies, suggesting the influence of associated deep or shallow trap levels within the bulk material [30]. The region associated with higher exponent are treated using equation (3) for exponential trap distribution.

In order to measure the electron mobility a sandwich structure consisting of ITO/ $\mathrm{Cs}_{2} \mathrm{CO}_{3} / \mathrm{P} 3 \mathrm{HT}: \mathrm{PCBM} / \mathrm{Al}$ is employed, where $\mathrm{Cs}_{2} \mathrm{CO}_{3}$ acts as a hole blocking layer. Corresponding dark $J-V$ characteristics is shown in Fig 1(b). Similar analysis for the range corresponding to the trap filled SCLC regime $\left(0.842 \mathrm{~V}<V_{\text {eff }}<1.43 \mathrm{~V}\right)$ reveals a value of electron mobility, $\mu_{e}=1.1 \times 10^{-8} \mathrm{~m}^{2} \mathrm{~V}^{-1} \mathrm{~s}^{-1}$. Initially the hole mobility reduces upon thermal annealing as is evident in Table 1 . However above 50 
${ }^{\circ} \mathrm{C}$ the reversal of this trend is observed with maximum hole mobility measured at $175{ }^{\circ} \mathrm{C}$. Mihailetchi et al [31] have previously reported on a similar observation of an increase in the hole mobility in the P3HT phase upon thermal annealing, with mobility values consistent with values measured in pristine P3HT $\left(\sim 10^{-8} \mathrm{~m}^{2} \mathrm{~V}^{-1} \mathrm{~s}^{-1}\right)$, i.e., in the absence of PCBM molecules. Furthermore, according to Yang et al [32], the improved value of the hole mobility is as a result of the recrystallization of P3HT phase. However in the case of the electron mobility, we have measured a reduction in this property at annealing temperature above $150^{\circ} \mathrm{C}$. The reason for this is somewhat unclear at this stage, although we suggest this might be due to the increased formation of PCBM aggregates, and also the extensive depletion regions around them, previously reported [23]. In other words, at very high annealing temperatures (i.e., above $150^{\circ} \mathrm{C}$ ), the resulting change in morphology, with its corresponding phase separation characterised by highly isolated PCBM aggregates does not facilitate electron transport. It is known that the charge transport in organic semiconductors is mainly due to hopping mechanism from site to site [33]. The increased separation between islands or clusters as shown in Fig. 3(c) may impede these hopping mechanisms lowering the value of electron mobility as recorded in our present study. Furthermore Fig. 4 indicates that the PCE peaks at $150^{\circ} \mathrm{C}$ and upon increasing the annealing temperature to $175^{\circ} \mathrm{C}$, a reduction in this parameter is observed. An important observation to note is that at $150^{\circ} \mathrm{C}$ where the maximum PCE was measured, the ratio of electron to hole mobility was determined to be 4.78. This observed trend, where the electron to hole mobility ratio reduces with annealing temperature, with a corresponding enhancement in PCEs (optimum at $150^{\circ} \mathrm{C}$ ) is consistent with previous work [28]. However, we have observed at $175^{\circ} \mathrm{C}$ lower than unity for this ratio $\left(\mu_{e} / \mu_{h} \sim\right.$ 0.357). This, we suggest is due to the disruption of electron transport pathways to the respective electrode as a result of PCBM clustering leading to lower electron mobility.

It is known that exciton dissociation rate and recombination rate, be it bimolecular or trap assisted are competing processes and increases as a result of improved charge carrier mobilities [24]. Due to the nature of fast charge transfer, photoluminescence measurements cannot elucidate on the recombination processes and instead we investigate photogeneration rate and exciton dissociation probability to identify dominant mechanism.

\subsection{Photocurrent generation rates and exciton dissociation probability}

Excitons created as a result of absorption of photons by polymer phase, may or may not result in dissociation of free charge carriers depending on the probability that these bound electron - hole pairs overcome their columbic attraction given by capture radius proposed by Onsager [34] 
$r_{\mathrm{c}}=\frac{q^{2}}{4 \pi \varepsilon_{\mathrm{r}} \varepsilon_{0} k_{\mathrm{B}} T}$

where $q$ is the electron charge, $\varepsilon_{\mathrm{r}}$ is the dielectric constant, $\varepsilon_{0}$ is the permittivity of free space, $k_{\mathrm{B}}$ is the Boltzmann constant and $T$ is the temperature. Due to low values of $\varepsilon_{r}$ associated with organic semiconductors; this capture radius is relatively larger than their inorganic counterparts. Furthermore this model suggests that the hot electron will impart its thermal energy by rapid motion settling at a distance $a$ from the hole, known as the thermalization length. The values of $a$ calculated on the basis of this model is around 2.5-3.5 nm which is larger than expected for nearest neighbouring charge transfer states $(1 \mathrm{~nm})$. To take into account of the relatively larger values of thermalization length, Braun [35] modified Onsager's model with the view that charge transfer states have a finite life time $\tau_{C T}$ which depends on the two competing mechanisms of dissociation and recombination as is given by Equation 5. This implies that the probability of exciton dissociation is dependent on the dissociation rate constant $k_{\mathrm{d}}(E, T, x)$ and the rate constant $k_{f}(T)$ associated with geminate recombination rate back to the ground state. This is expressed as

$$
P(E, T, x)=\frac{k_{\mathrm{d}}}{k_{\mathrm{d}}+k_{f}}=k_{\mathrm{d}} \tau_{\mathrm{CT}}
$$

It is worth noting that when $k_{\mathrm{d}}>>k_{f}$ the probability approaches unity and the charge transfer state life time is described by the dissociation rate, while at the other extreme when $k_{f}>k_{\mathrm{d}}$ the probability approaches zero and the charge transfer states life time will approach the geminate recombination life time back to the ground state. The dissociation rate $k_{\mathrm{d}}$ is given by

$$
k_{\mathrm{d}}(E, T, x)=\frac{3 k_{\mathrm{r}}}{4 \pi a^{3}} \exp \left(-\frac{\Delta E}{k_{\mathrm{B}} T}\right)\left[1+b+\frac{b^{2}}{3}+\frac{b^{3}}{18}+\frac{b^{4}}{180}+\ldots\right]
$$

where $k_{\mathrm{r}}$ is the recombination constant (either Langevin $\left(B_{L}\right)$ or trap assisted recombination referred to as Shockley Read Hall $\left.\left(B_{\mathrm{SRH}}\right)\right), a$ is the thermalization length or initial hot electron and hole pair separation, $k_{\mathrm{B}}$ is the Boltzmann constant, $T$ is the temperature, $b=\frac{q^{3} E}{8 \pi\left(\varepsilon_{\mathrm{r}}\right) \varepsilon_{0} a^{3}}$ and $\Delta E$ is the binding energy for the electron-hole pair given by

$\Delta E=\frac{q^{2}}{4 \pi\left\langle\varepsilon_{\mathrm{r}}\right\rangle \varepsilon_{0} a}$

For Langevin recombination constant, $B_{L}$ may be presented as

$$
B_{\mathrm{L}}=\frac{\langle\mu\rangle q}{\varepsilon_{0}\left\langle\varepsilon_{\mathrm{r}}\right\rangle}
$$


where $\langle\mu\rangle$ is the average sum of the electron and hole mobilities. The photocurrent generation [31] is given by

$$
J_{\mathrm{ph}}=P(E, T, x) G_{\max } q d
$$

where $G_{\max }$ is the maximum generation rate, $q$ is the electronic charge and $d$ is the active layer thickness. Based on Langevin model and the values of the electron and hole mobilities obtained in section 3.1, the rate constant $k_{f}$ associated with geminate recombination back to ground state is calculated as $k_{f}=1 \times 10^{4} \mathrm{~s}^{-1}\left(B_{\mathrm{L}}=6.11 \times 10^{-18} \mathrm{~m}^{3} \mathrm{~s}^{-1}\right) \quad$ in agreement with previous report [36]. Upon thermal annealing at $150{ }^{\circ} \mathrm{C}$, the rate of decay $k_{f}$ increases to $2 \times 10^{4} \mathrm{~s}^{-1}\left(B_{\mathrm{L}}=1.22 \times 10^{-17} \mathrm{~m}^{3} \mathrm{~s}^{-1}\right)$. Further thermal annealing at $175^{\circ} \mathrm{C}$, results in further increase of this parameter to $8 \times 10^{4} \mathrm{~s}^{-1}\left(B_{\mathrm{L}}=5.64 \times 10^{-17}\right.$ $\left.\mathrm{m}^{3} \mathrm{~s}^{-1}\right)$. This increase in decay rate is attributed to the increase in hole carrier mobility, resulting in a higher value of Langevin constant $B_{L}$ and faster geminate recombination back to the ground state corresponding to a shorter charge transfer state life time. Similarly for trap assisted recombination, $B_{\mathrm{SRH}}$ is given as [26]

$$
B_{\mathrm{SRH}}=\frac{C_{n} C_{p} N_{\mathrm{T}_{\mathrm{e}}}}{\left[C_{n}\left(n+n_{1}\right)+C_{p}\left(p+p_{1}\right)\right]}
$$

where $C_{n}$ is defined as the probability per unit time that an electron in PCBM phase is captured by an empty trap. Similarly $C_{p}$ is defined as the probability per unit time that a hole is captured by an electron- occupied trap site, $N_{\mathrm{T}_{\mathrm{e}}}$ is the density of the electron traps, $n$ and $p$ are the electron and hole density in the conduction and valence band respectively, and $n_{1} p_{1}=n_{i}^{2}$ the intrinsic carrier concentrations. Intrinsic carrier concentrations are much lower than electron and hole densities and therefore equation 10 can be reduced to

$$
B_{\mathrm{SRH}}=\frac{C_{n} C_{p} N_{\mathrm{T}_{\mathrm{e}}}}{\left[C_{n}(n)+C_{p}(p)\right]}
$$

Assuming $C_{n}=C_{p}$ equation (11) can be simplified as;

$$
B_{\mathrm{SRH}}=\frac{C_{n} N_{\mathrm{T}_{\mathrm{e}}}}{n+p}
$$

Based on this model a double logarithmic plot of photocurrent $\left(J_{\mathrm{ph}}=J_{\text {light }}-J_{\text {dark }}\right)$ versus the effective reverse applied voltage $\left(V_{0}-V\right)$, where $V_{0}$ is the voltage at which $J_{\mathrm{ph}}=0$, for as cast and thermally annealed at $150{ }^{\circ} \mathrm{C}$ is presented in Fig. 5. Using the data provided in Table 1 and assuming a value for $C p=1.6 \times 10^{-18} \mathrm{~m}^{3} \mathrm{~s}^{-1}$, equation 12 yields a value of $B_{\mathrm{SRH}}=2 \times 10^{-16} \mathrm{~m}^{3} \mathrm{~s}^{-1}$ which subsequently leads to $k_{f}$ $=2.3 \times 10^{5} \mathrm{~s}^{-1}$ for as cast and at $150{ }^{\circ} \mathrm{C}$ a value of $B_{\mathrm{SRH}}=2.5 \times 10^{-15} \mathrm{~m}^{3} \mathrm{~s}^{-1}$ with corresponding $k_{f}=2 \times 10^{6}$ 
$\mathrm{s}^{-1}$. Further increase in the annealing temperature to $175^{\circ} \mathrm{C}$ resulted in a reduction of $B_{\mathrm{SRH}}$ to $1.06 \times 10^{-}$ ${ }^{16} \mathrm{~m}^{3} \mathrm{~s}^{-1}$ with corresponding $k_{f}=6 \times 10^{4} \mathrm{~s}^{-1}$, as is expected from the reduction in the value of the trap density. The higher recombination constant $B_{\mathrm{SRH}}$ in comparison to $B_{\mathrm{L}}$ is an indication that the trap assisted recombination is dominant in comparison to its Langevin counterpart. The highest PCEs measured in the present work correspond to thermal annealing at $150^{\circ} \mathrm{C}$. Therefore on the basis of this, a lower recombination rate should be expected. On the contrary this is not so, as the results indicates higher recombination constant with higher geminate recombination rate back to the ground state $k_{f}$. Referring again to Table 1 it is noticed that the trap concentration has increased by almost 2 orders of magnitude which resulted in a higher value of $B_{\mathrm{SRH}}$. However, the improved ratio of electron/hole mobility (Table 1) upon thermal annealing plays an important role in charge transport to the respective electrodes, hence improving PCE. In order to further verify the dominant process considered above, we refer to the relationship between light intensity and open circuit voltage given by [36].

$V_{\mathrm{OC}}=\frac{E_{\mathrm{g}}}{q}-\frac{k_{\mathrm{B}} T}{q} \ln \left(\frac{(1-P) B_{L} N_{\mathrm{CV}}^{2}}{P G}\right)$

where $E \mathrm{~g}$ is the energy gap or the difference between the LUMO and HOMO levels of PCBM and $\mathrm{P} 3 \mathrm{HT}, k_{\mathrm{B}}$ is the Boltzmann constant, $T$ is the temperature, $q$ is the electronic charge, $P$ is the probability of exciton dissociations, $B_{L}$ is the Langevin recombination constant, and $N_{\mathrm{CV}}$ is the effective density of states in the conduction or valence band. In the event were traps are absent the above relationship holds with slope equalling $k_{\mathrm{B}} T / q(\mathrm{eV})$, when $V_{\mathrm{OC}}$ is plotted as a function of the logarithm of light intensity. The higher value of the slope coefficient is associated with the strength of trap-assisted recombination. Fig. 6 shows a semi $\log$ plot of $V_{\mathrm{OC}}$ versus the light intensity. As is evident, the slope of $1.12\left(k_{\mathrm{B}} T / q\right)$ is indicative of trap assisted recombination. Upon thermal annealing at $150{ }^{\circ} \mathrm{C}$ the value of this slope increased to $1.46\left(k_{B} T / q\right)$ indicating an increase in the strength of the trap assisted recombination. This correlates well with the increase in trap density calculated in section 3.1 .

\section{Conclusions}

Previously it was reported that a system consisting of a highly intermixed blend of PCBM/P3HT could result in additional disorder and formations of traps [30]. Our results support this view in the light of high trap densities measured. Furthermore the increase in electron trap density upon thermal annealing correlates with glass transition temperature for P3HT phase [25] initiating traps formation in the heterojunction device. The ratio of electron to hole mobility improves with annealing temperature up 
to $150{ }^{\circ} \mathrm{C}$ above which the hole mobility overtakes electron mobility resulting from segregation of PCBM and formation of clusters and disruption of the transport path ways for electrons and deterioration of the PCEs. The higher recombination constant $B_{\mathrm{SRH}}$ measured in this investigation in comparison to $B_{\mathrm{L}}$ is an indication that the trap assisted recombination is dominant in comparison to its Langevin counterpart. The presence of high density of traps in this blended material further supports the Shockley-Read-Hall trap assisted recombination with further evidence of the dependence of open circuit voltage on light intensity.

\section{References}

[1] S. R. Forrest, M. E. Thompson, Chem. Rev. 107 (2007) 923-925.

[2] F. C. Krebs, R. Søndergaard, M. Jørgensen, Solar Energy Materials and Solar Cells, 95 (2011) 1348-1353.

[3] F.C. Krebs, Fabrication and processing of polymer solar cells, A review of printing and coating techniques, Solar Energy Materials and Solar Cells, 93 (2009) 394-412.

[4] F.C. Krebs, K. Norrman, Using light induced thermocleavage in a roll-to- roll process for polymer solar cells, ACS Appl. Mater. Interfaces 2 (2010) 877-887.

[5] M. Campoy-Quiles, T. Ferenczi, T. Agostinelli, P. G. Etchegoin, Y. Kim, T. D. Anthopoulos, P. N. Stavrinou, D. D. C. Bradley, J. Nelson, Morphology evolution via self-organization and lateral and vertical diffusion in polymer:fullerene solar cell blend, Nature Materrials 7 (2008) 158-164.

[6] F-C. Chen, C-J. Ko, J-L. Wu, W-C. Chen, Morphological study of P3HT: PCBM blend films prepared through solvent annealing for solar cell applications, Solar Energy Materials and Solar Cells, 94 (2010) 2426-2430.

[7] G. Kalita, M. Masahiro, W. Koichi, M. Umeno, Solid-State Electronics, 54 (2010) 447-451. 
[8] K. S. Nalwa, H. K. Kodali, B. Ganapathysubramnian, Dependence of recombination mechanisms and strength on processing conditions in polymer solar cells, Applied Physics Letters, 99, (2011) $263301,1-3$.

[9] R. F. Stephen, The Limits to Organic Photovoltaic Cell Efficiency, MRS Bulletin 30 (2005) 28-32.

[10] H. Spanggaard, F. C. Krebs, A brief history of the development of organic and polymeric photovoltaics, Solar Energy Materials and Solar Cells, 83 (2004) 125-146.

[11] G. Li, R. Zhu, Y. Yang, Polymer Solar Cells, Nature Photonics, 6 (2012) 153-161.

[12] A. C. Mayer, S. R. Scully, B. E. Hardin, M. W. Rowell, M. D. McGehee, Polymer-based solar cells, Materials Today 10 (2007) 28-33.

[13] M. C. Scharber, N. S. Sariciftci, Efficiency of Bulk-Heterojunction Organic Solar Cells, Progress in Polymer Science (2013), http://dx.doi.org/10.1016/j.progpolymsci.2013.05.001.

[14] H. Hoppe, M. Niggemann, C. Winder, J. Kraut, R. Hiesgen, A. Hinsch, D. Meissner, N. S. Saciciftci, Nanoscale Morphology of Conjugated Polymer/Fullerene-Based Bulk-Heterojunction Solar Cells, Advanced Functional Materials, 14 (2004) 1005-1011.

[15] A. Pivrikas, H. Neugebauer, N. S. Sariciftci, Influence of processing additives to nanomorphology and efficiency of bulk-heterojunction solar cells: A comparative review, Solar Energy, 85 (2011) 1226-1237.

[16] K. Kim, J. Liu, M. A. G. Namboothiry, D. L. Carroll, Roles of donor and acceptor nanodomains in 6\% efficient thermally annealed polymer photovoltaics, Applied Physics Letters, 90 (2007) 163511, 1-3.

[17] V.D. Milhailetchi, H. Xie, B. De Boer, L. M. Popescu, J. C. Hummelen, P. W. M. Blom, Origin of the enhanced performance in poly(3-hexylthiophene): [6,6]-phenyl $\mathrm{C}_{61}$-butyric acid methyl ester solar cells upon slow drying of the active layer, Applied Physics Letters, 89 (2006) 012107, 1-3.

[18] L. J. A. Koster, V.D. Milhailetchi, P. W. M. Blom, Bimolecular recombination in polymer/fullerene bulk heterojunction solar cells, Applied Physics Letters, 88 (2006) 052104, 1-3.

[19] S. Miller, G. Fanchini, L. Yun-Yue, L. Cheng, C. Chun-Wei, S. Wei-Fang, C. Manish, Investigation of nanoscale morphological changes in organic photovoltaics during solvent vapour annealing, Journal of Material Chemistry 18 (2008) 306-312. 
[20] Z. Zhuo. F. Zang, J. Wang, J. Wang, X. Xu, Z. Xu, Y. Wang, Efficiency improvement of polymer solar cells by iodine doping, Solid-State Electronics, 63 (2011) 83-88.

[21] J. Peet, J. Y. Kim, N. E. Coates, W. L. Ma, D. Moses, A. J. Heeger, G. C. Bazan, Efficiency enhancement in low-bandgap polymer solar cells by processing with alkane dithiols, Nature Materials, 6 (2007) 497-500.

[22] Y. Sun, J. Liu, Y. Ding, Y. Han, Decreasing the Aggregation of PCBM in P3HT/PCBM Blend Films by Cooling the Solution, Colloids and Surfaces A: Physicochemical and Engineering Aspects, 421 (2013) 135-141.

[23] O. Oklobia, T. S. Shafai, A quantitative study of the formation of PCBM clusters upon thermal annealing of P3HT/PCBM bulk heterojunction solar cell, Solar Energy Materials and Solar Cells, 117 (2013) 1-8.

[24] T. M. Clarke, J. R. Durrant, Charge Photogeneration in Organic Solar Cells, Chemical Review, 110 (2010) 6736-6767.

[25] Y. Kim, S. A. Choulis, J. Nelson, D. D. C. Bradley, Device annealing effect in organic solar cells with blends of poly (3-hexylthiophene) and soluble fullerene, Applied Physics Letters, 86 (2005) 063502, 1-3.

[26] K. S. Nalwa, R. C. Mahadevapuram, S. Chaudhary, Growth rate dependent trap-density in polythiophene-fullerene solar cells and its implications, Applied Physics Letters, 98 (2011) 093306, 1-3.

[27] V. Shrotriya, Y. Yao, G. Li, Y. Yang, Effect of self-organization in polymer/fullerene bulk heterojunctions on solar cell performance, Applied Physics Letters, 89 (2006) 063505, 1-3.

[29] M. A. Lampert, P. Mark, Current Injection in Solids, Academic, New York 1970.

[30] C-Y. Nam, D. Su, C.T. Black, High-Performance Air-Processed Polymer-Fullerene Bulk Heterojunction Solar Cells, Advanced Functional Materials, 19 (2009) 3552-3559.

[31] V. D Mihailetchi, H. Xie, D. de Boer, L. J. A. Koster, P. W. M. Blom, Charge Transport and Photocurrent Generation in Poly (3-hexylthiophene): Methanofullerene Bulk-Heterojunction Solar Cells, Advanced Functional Materials, 16 (2006) 699-708. 
[32] X. Yang, J. Loos, S. C. Veenstra, W. J. H. Verhees, M. M. Wienk, J. M. Kroon, M. A. J. Michels, R. A. J. Janssen, Nano Letters, 5 (2005), 579-583.

[33] S. Peter, Electrical Characterisation of Organic Electronic Materials and Devices, Wiley, New Jersey 2009.

[34] L. Onsager, Initial Recombination of Ions, Physical Review, 54 (1938) 554-557.

[35] C. L. Braun, Electric field assisted dissociation of charge transfer states as a mechanism of photocarrier production, Journal of Chemical Physics 80 (1983) 1-5.

[36] L. J. A. Koster, V. D Mihailetchi, P. W. M. Blom, Bimolecular recombination in polymer/fullerene bulk heterojunction solar cells, Applied Physics Letters, 88 (2006) 052104, 1-3. 


Fig. 1: Dark $J-V$ characteristics of P3HT: PCBM blend: (a) Hole-only device (reversed biased), and (b) Electron-only device (forward biased). The inserts show the fits of experimental data to the SCLC model in equation 2 .
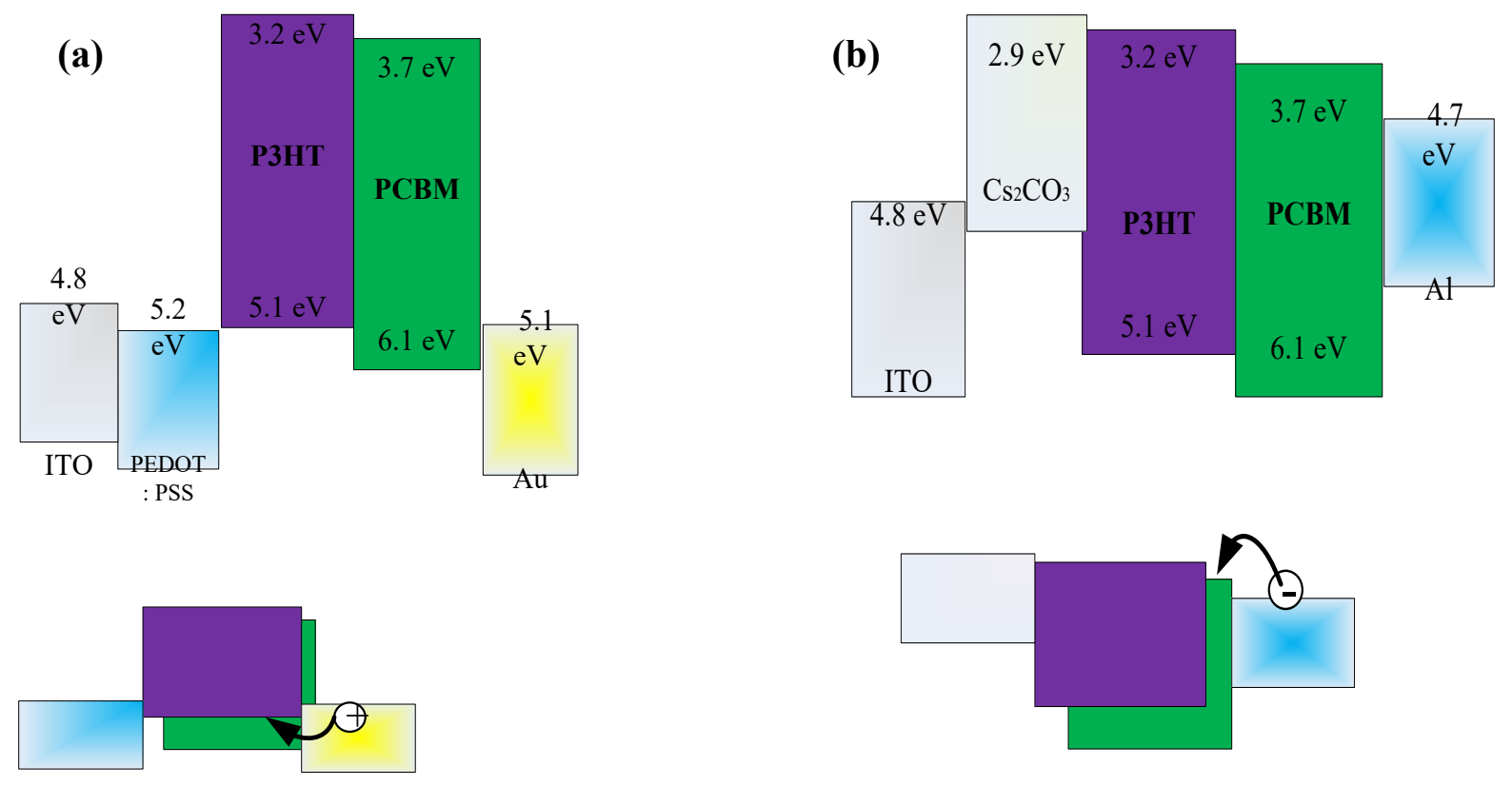

Fig. 2: Schematic energy band diagram for (a) hole-only device and (b) electron-only device 
(a)

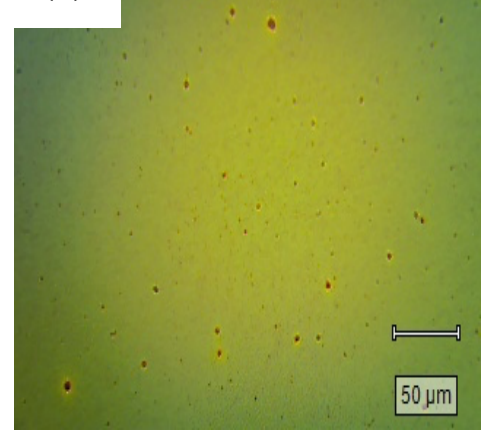

(b)

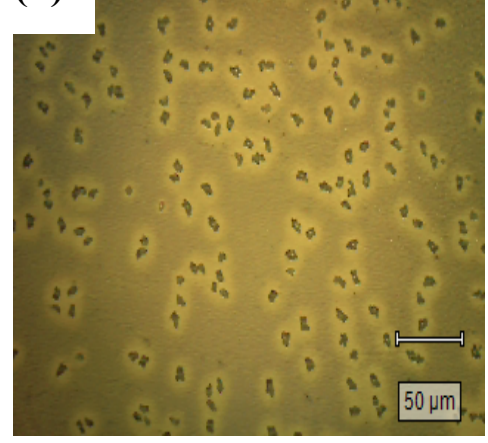

(c)

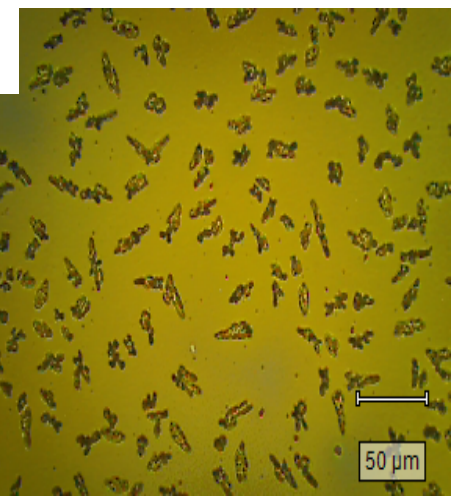

Fig. 3: Optical microscopic images of films of thickness of $\sim 150 \mathrm{~nm}$; (a) as-cast, after thermal annealing at (b) $150^{\circ} \mathrm{C}$ and (c) $175^{\circ} \mathrm{C}$

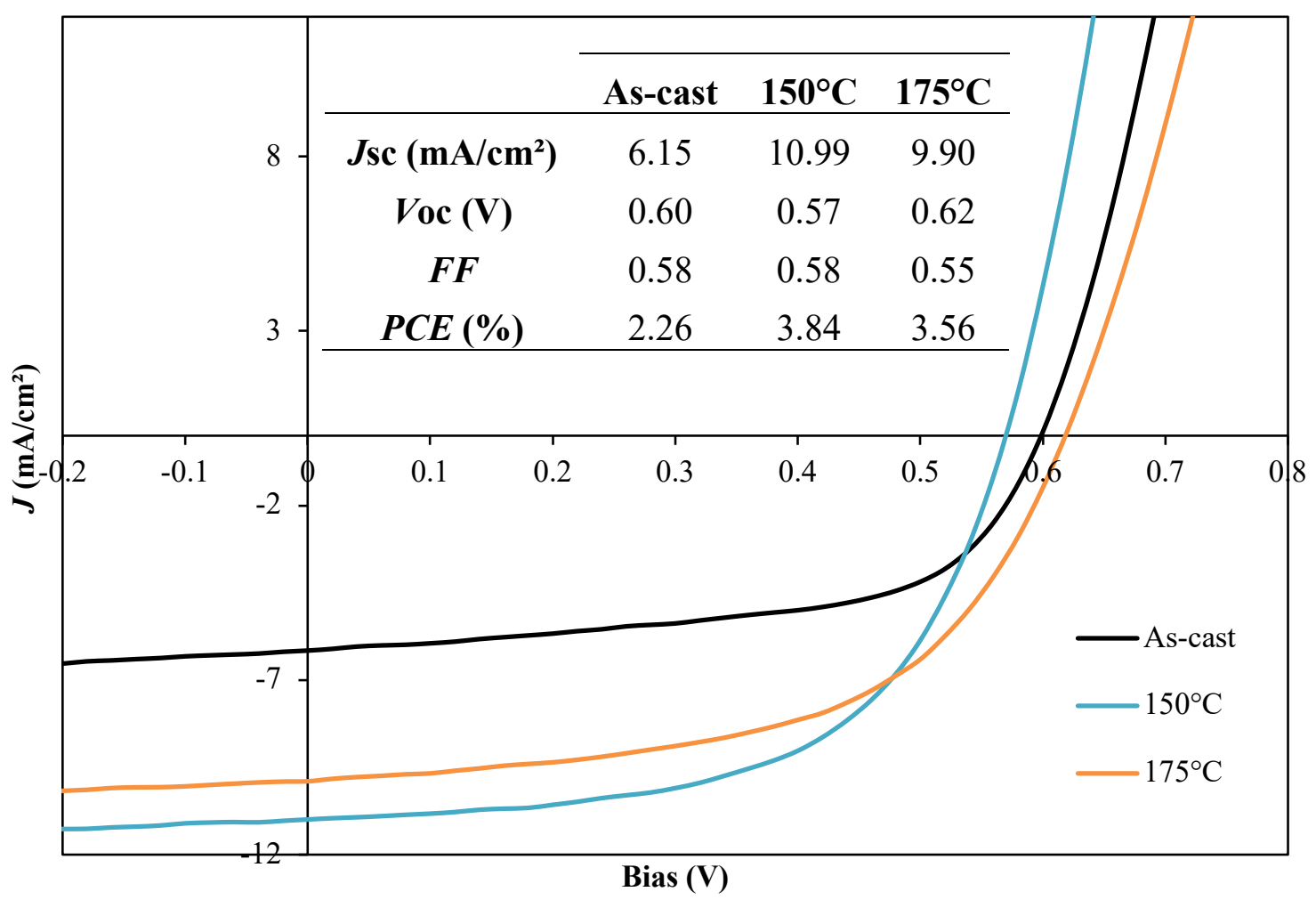

Fig. 4: $J-V$ characteristics of P3HT: PCBM blend device based on an active layer thickness of $\sim 150$ $\mathrm{nm}$; as-cast and after annealing at $150^{\circ} \mathrm{C}$ and $175^{\circ} \mathrm{C}$, for 10 minutes each. Insert shows the summary of device performance parameters 



Fig. 5: Photocurrent density $\left(J_{\mathrm{ph}}\right)$ as a function of effective reverse applied voltage $\left(V_{0}-V\right)$ for P3HT: PCBM based solar cell device; (a) as-cast and (b) after thermal annealing at $150^{\circ} \mathrm{C}$. The solid lines represent numerical calculations based on Shockley-Read-Hall (blue), and Langevin (broken, red) recombination. 


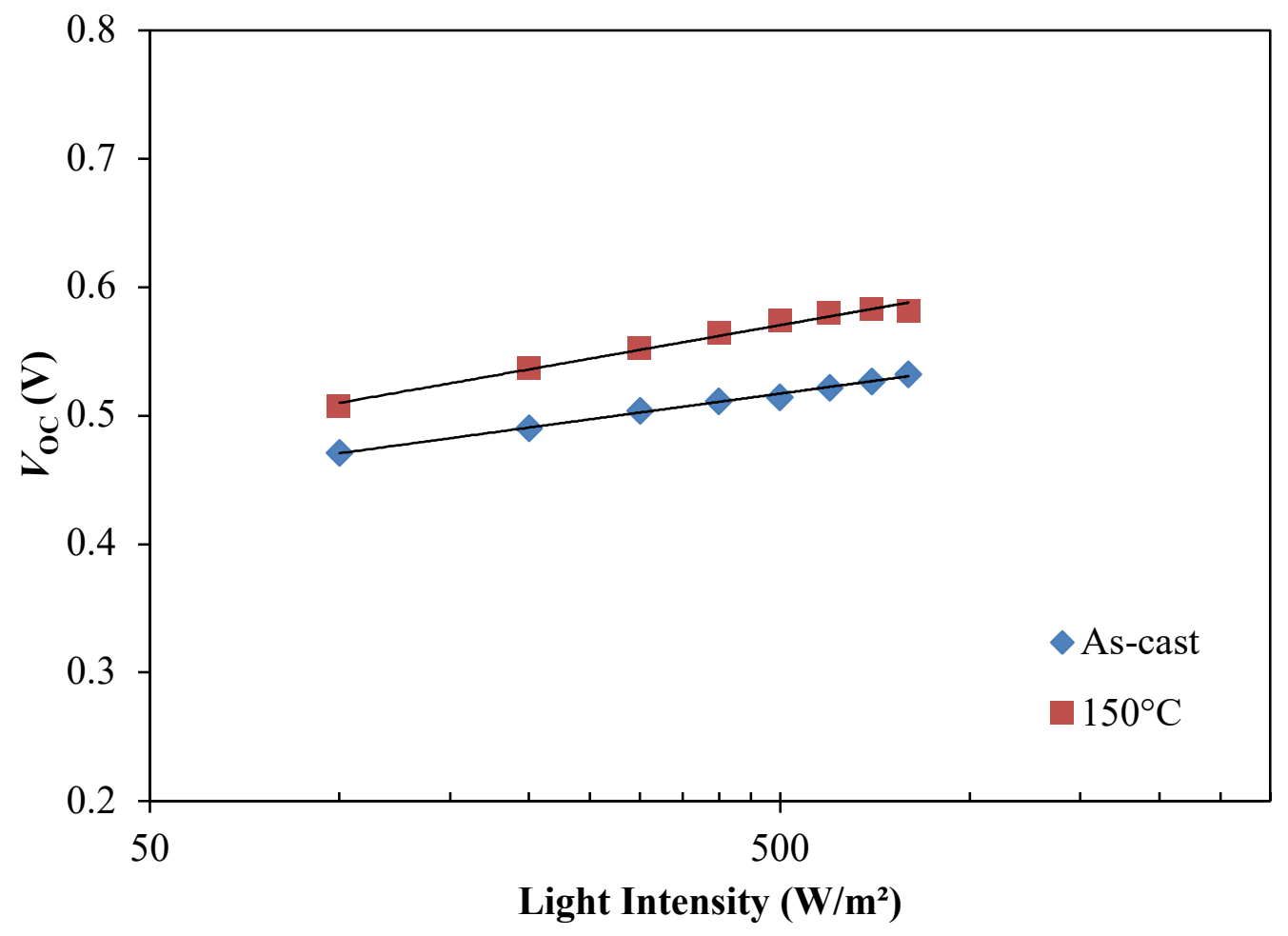

Fig. 6: $V_{\mathrm{OC}}$ as a function of light intensity for P3HT: PCBM based solar cell device; as-cast and annealed at $150^{\circ} \mathrm{C}$, solid lines are linear fits used to determine the slopes. 


\begin{tabular}{|c|c|c|c|c|c|c|}
\hline & As cast & $5^{\circ}{ }^{\circ} \mathrm{C}$ & $100^{\circ} \mathrm{C}$ & $125^{\circ} \mathrm{C}$ & $150^{\circ} \mathrm{C}$ & $175^{\circ} \mathrm{C}$ \\
\hline$\mu_{h}\left(\mathrm{~m}^{2} \mathrm{~V}^{-1} \mathrm{~s}^{-1}\right)$ & $1.15 \times 10^{-9}$ & $6.68 \times 10^{-10}$ & $8.53 \times 10^{-10}$ & $2.14 \times 10^{-9}$ & $2.30 \times 10^{-9}$ & $1.06 \times 10^{-8}$ \\
\hline$\mu_{e}\left(\mathrm{~m}^{2} \mathrm{~V}^{-1} \mathrm{~s}^{-1}\right)$ & $1.1 \times 10^{-8}$ & $5.93 \times 10^{-9}$ & $8.51 \times 10^{-9}$ & $1.31 \times 10^{-8}$ & $1.1 \times 10^{-8}$ & $3.78 \times 10^{-9}$ \\
\hline$\mu_{e} / \mu_{h}$ & 9.56 & 8.88 & 9.98 & 6.12 & 4.78 & 0.357 \\
\hline$n\left(\mathrm{~m}^{-3}\right)$ & $4.9 \times 10^{21}$ & $4.74 \times 10^{21}$ & $7.09 \times 10^{22}$ & $6.37 \times 10^{22}$ & $6.05 \times 10^{22}$ & $2.65 \times 10^{21}$ \\
\hline$p\left(\mathrm{~m}^{-3}\right)$ & $1.49 \times 10^{22}$ & $1.06 \times 10^{22}$ & $9.68 \times 10^{21}$ & $1.08 \times 10^{22}$ & $1.17 \times 10^{22}$ & $6.22 \times 10^{22}$ \\
\hline$N_{T h}\left(\mathrm{~m}^{-3}\right)$ & $2.98 \times 10^{25}$ & $6.79 \times 10^{24}$ & $2.22 \times 10^{24}$ & $7.72 \times 10^{24}$ & $1.36 \times 10^{23}$ & $1.14 \times 10^{26}$ \\
\hline$N_{T e}\left(\mathrm{~m}^{-3}\right)$ & $2.51 \times 10^{24}$ & $6.76 \times 10^{24}$ & $2.54 \times 10^{26}$ & $3.79 \times 10^{26}$ & $1.14 \times 10^{26}$ & $4.30 \times 10^{24}$ \\
\hline $\begin{array}{l}\text { Ohmic region* } \\
\text { (V) }\end{array}$ & $0.20-0.70$ & $0.25-0.60$ & $0.35-0.80$ & $0.45-0.95$ & $0.45-0.95$ & $0.50-1.00$ \\
\hline $\begin{array}{l}\text { SCLC region* } \\
\text { (V) }\end{array}$ & $3.45-4.35$ & $1.85-3.00$ & $1.25-2.20$ & $1.45-2.45$ & $1.70-2.40$ & $2.30-3.15$ \\
\hline $\begin{array}{l}\text { Trap-limited } \\
\text { region* }(V)\end{array}$ & $5.25-6.75$ & $5.00-6.15$ & $4.50-5.95$ & $3.00-6.45$ & $4.60-6.90$ & $3.50-6.45$ \\
\hline $\begin{array}{l}\text { Ohmic region }^{\dagger} \\
\text { (V) }\end{array}$ & $0.10-0.43$ & $0.1-0.39$ & $0.15-0.38$ & $0.10-0.70$ & $0.15-0.65$ & $0.10-0.55$ \\
\hline $\begin{array}{l}\text { SCLC region }^{\dagger} \\
\text { (V) }\end{array}$ & $0.84-1.43$ & $0.78-1.21$ & $2.00-2.48$ & $2.80-3.90$ & $4.80-5.95$ & $1.88-2.72$ \\
\hline $\begin{array}{l}\text { Trap-limited } \\
\text { region }^{\dagger}(\mathrm{V})\end{array}$ & $1.51-2.22$ & $1.76-2.21$ & $2.48-2.73$ & $3.85-4.45$ & $5.80-6.45$ & $3.13-4.13$ \\
\hline
\end{tabular}

* Hole - only devices, $\dagger$ Electron - only devices: Voltage ranges

Table 1: Summary of the parameters determined from experimental data. 